\title{
Le Concours Science \& Humour 2017 de la SFP
}

\author{
Montrer la science et/ou la vie des chercheurs et chercheuses \\ sur un ton décalé et humoristique : voilà quel était le thème \\ de ce concours d'images (dessins, bandes dessinées, photos), \\ lancé par la Société Française de Physique dans le cadre \\ de son $24^{\mathrm{e}}$ Congrès général.
}

Parce que la science rime aussi avec amusement, créativité et enthousiasme, le Concours Science \& Humour s'est donné pour objectif de valoriser l'aspect ludique et dynamique associé à la démarche scientifique. Par l'intermédiaire de l'image et de l'humour, il a également offert aux scientifiques l'occasion de montrer leur quotidien et les enjeux de leurs métiers passionnants.

Ouvert aux scientifiques en herbe comme aux confirmés, le concours Science \& Humour a été parrainé par le mathématicien Cédric Villani, médaille Fields 2010, et soutenu par de nombreux partenaires (voir la liste p. 37). Les candidats ont eu jusqu'au 2 mai 2017 pour déposer leurs œuvres sur le site du congrès www.sfp2017.fr.
En moins de trois mois, près de 200 propositions d'une surprenante variété ont été reçues : bandes dessinées, schémas, dessins et photographies, envoyés par des passionnés de tous âges et de toutes provenances. De l'élève de primaire au chercheur et à la chercheuse émérite, en passant par la secrétaire médicale, lejuriste, l'enseignant(e), l'ingénieur(e), l'archéologue ou le kinésithérapeute, les artistes et amateurs de science se trouvent partout et ce concours nous l'a montré. Pari réussi donc!

Le jury, composé des partenaires de l'opération, a sélectionné sept œuvres lauréates, dont la reproduction se trouve ci-après. Un prix du public a également été décerné, après consultation par voie électronique, une fois le concours clos.
Quand Sciences \& Avenir en parle: "Les lauréats du concours

Science \& Humour de la Société

Française de Physique ", article à lire sur www.sciencesetavenir.fr

\section{Les lauréats}

Catégorie Dessin

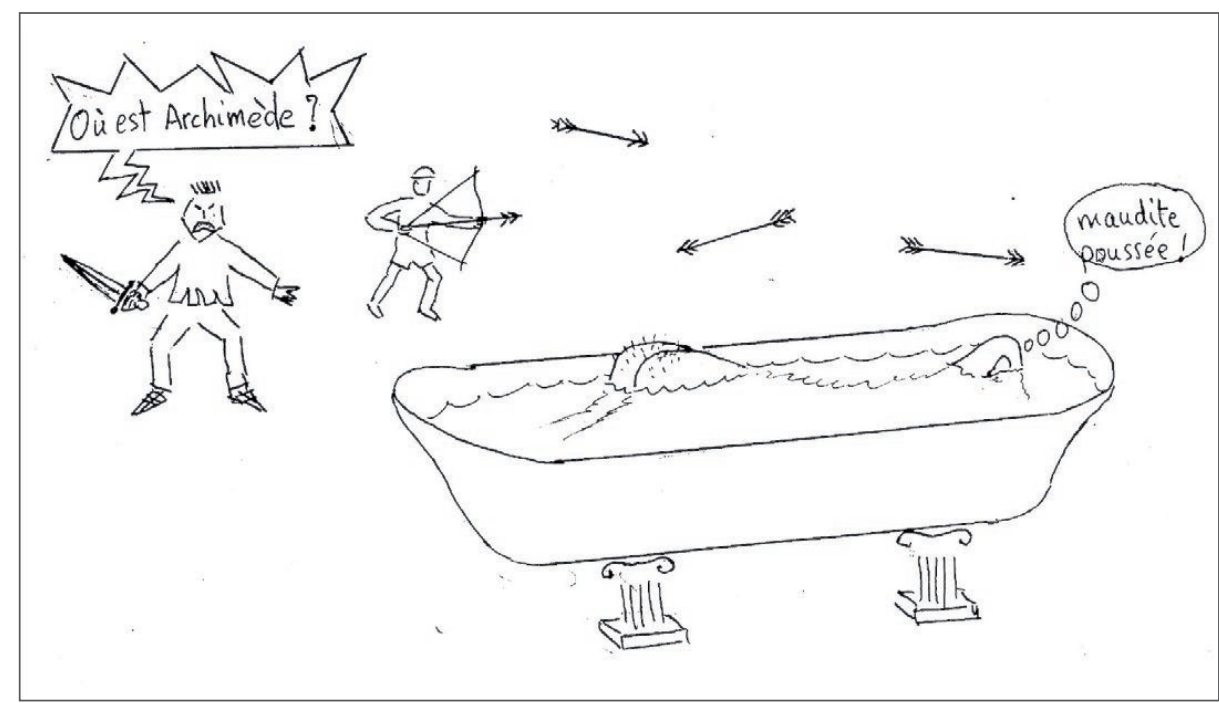

Titre : Archimède. Auteur : Xavier Artru - 75 ans - Sainte-Foy-lès-Lyon.

Xavier est directeur de recherche émérite CNRS à l'Institut de Physique Nucléaire de Lyon. Après avoir été président de la section locale Rhône de la SFP, il en est actuellement le trésorier. 


\section{Catégorie Photo}

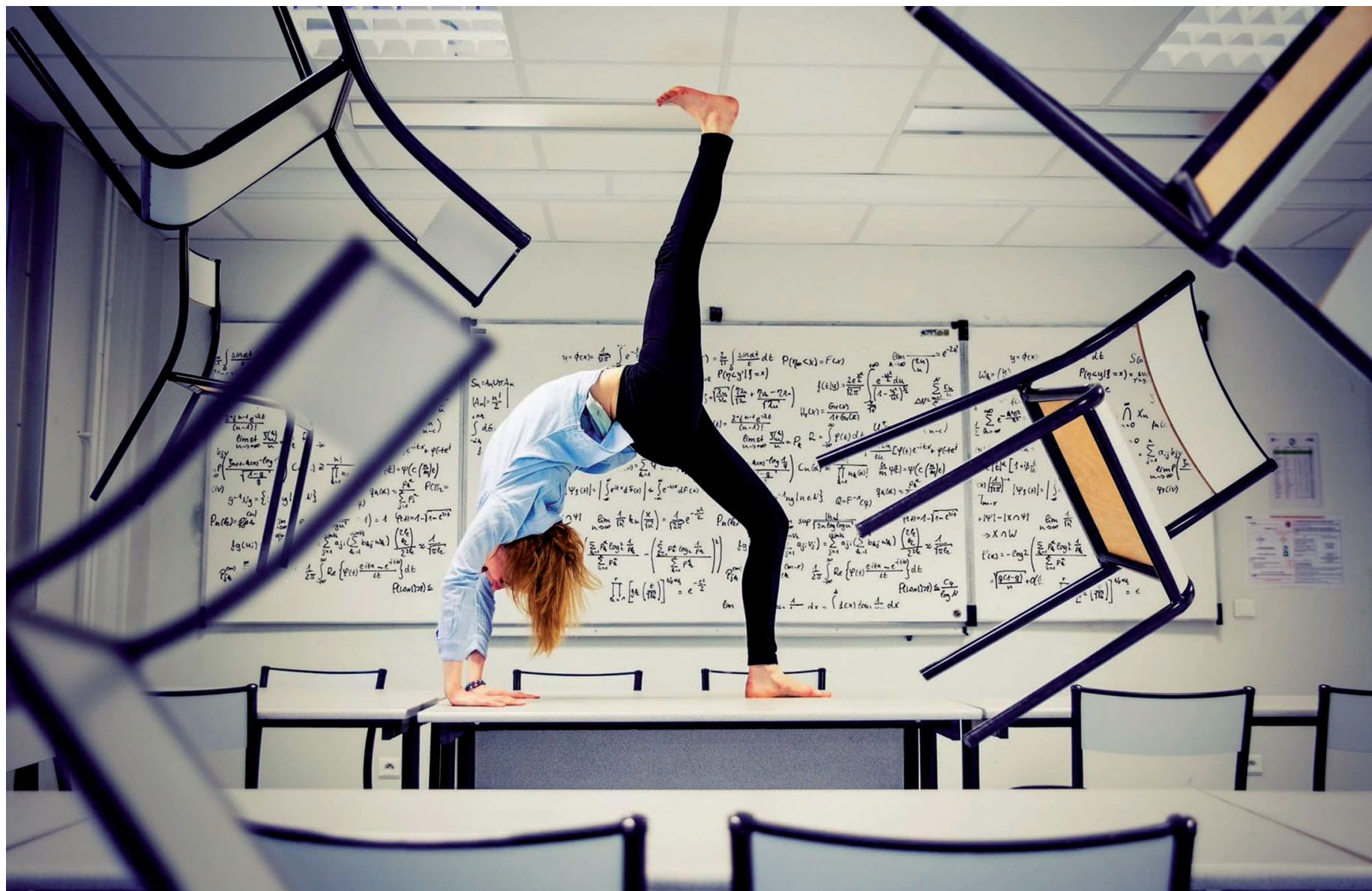

Titre : Faire preuve de souplesse pour adopter le meilleur point de vue. Auteurs : Orane Tasky et Déborah Michel - 25 ans toutes les deux - Seine-Maritime. Orane, graphiste salariée, développe la photo à son compte (orane-taskywixsite.com/), et Déborah est doctorante en mathématiques au Laboratoire de Mathématiques Raphaël Salem (Université de Rouen).

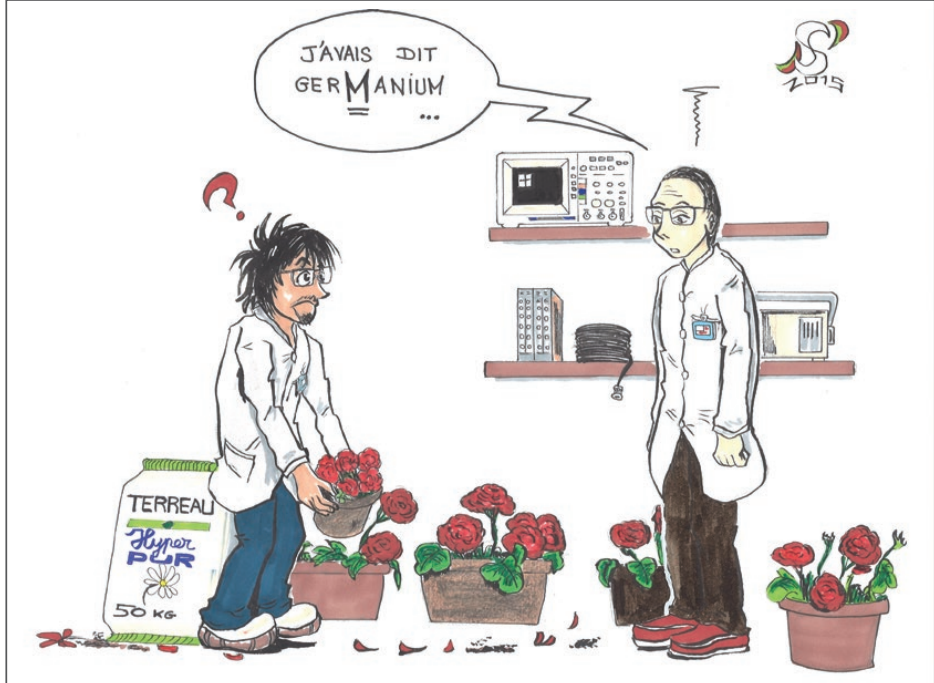

Titre : GerManium. Auteur : Benoît Simony - 26 ans - Aix-en-Provence. Benoît est doctorant en troisième année au Laboratoire de Mesures Nucléaires (LMN) du CEA Cadarache.

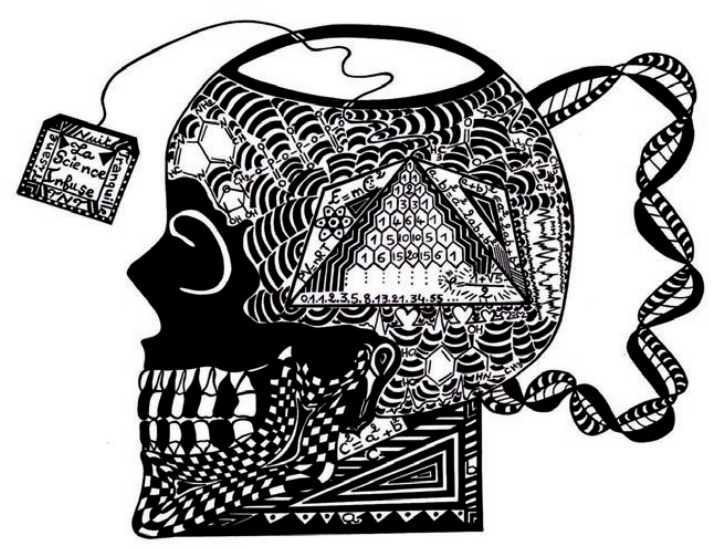

LA SCIENCE INFUSE

27

Titre : La Science infuse. Auteur : Mohammed Moudjou - 56 ans Draveil (91).

Mohamed est ingénieur de recherche en biologie à I'INRA de Jouyen-Josas. Cette œuvre a été réalisée au stylo BIC noir. 
Catégorie BD

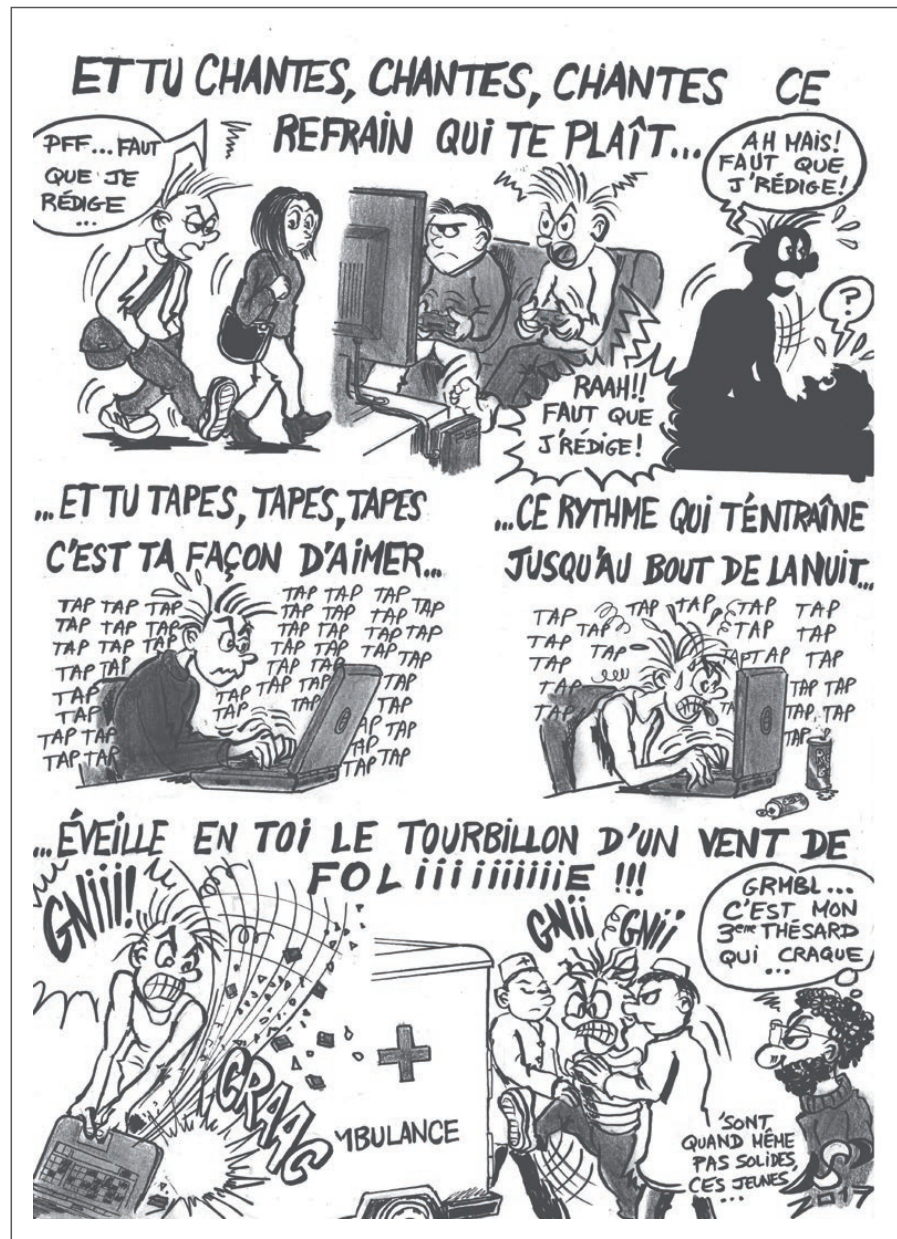

Titre : Nuit de rédac. Auteur : Benoît Simony. Benoît a aussi été primé en catégorie « dessin ».

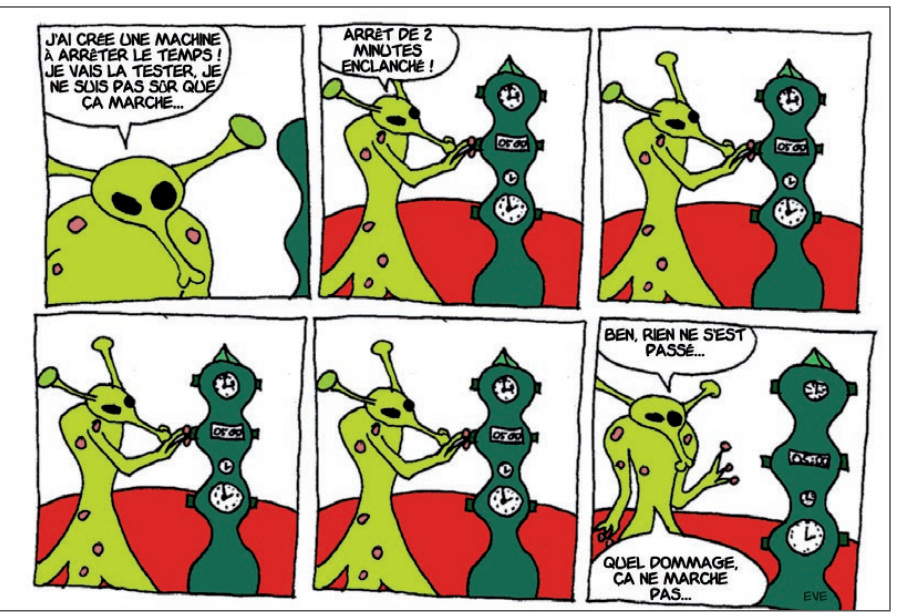

Titre : Zblorg \& Krakbuk « Le temps passe vite». Auteur : Ève Redero 28 ans - Paris. Ėve est ingénieure en électronique chez Devialet.
Prix du public

Un vote du public, auquel plus de 600 personnes ont participé, a élu La Cuisine, dessin/bd de Zélie Tournoud.
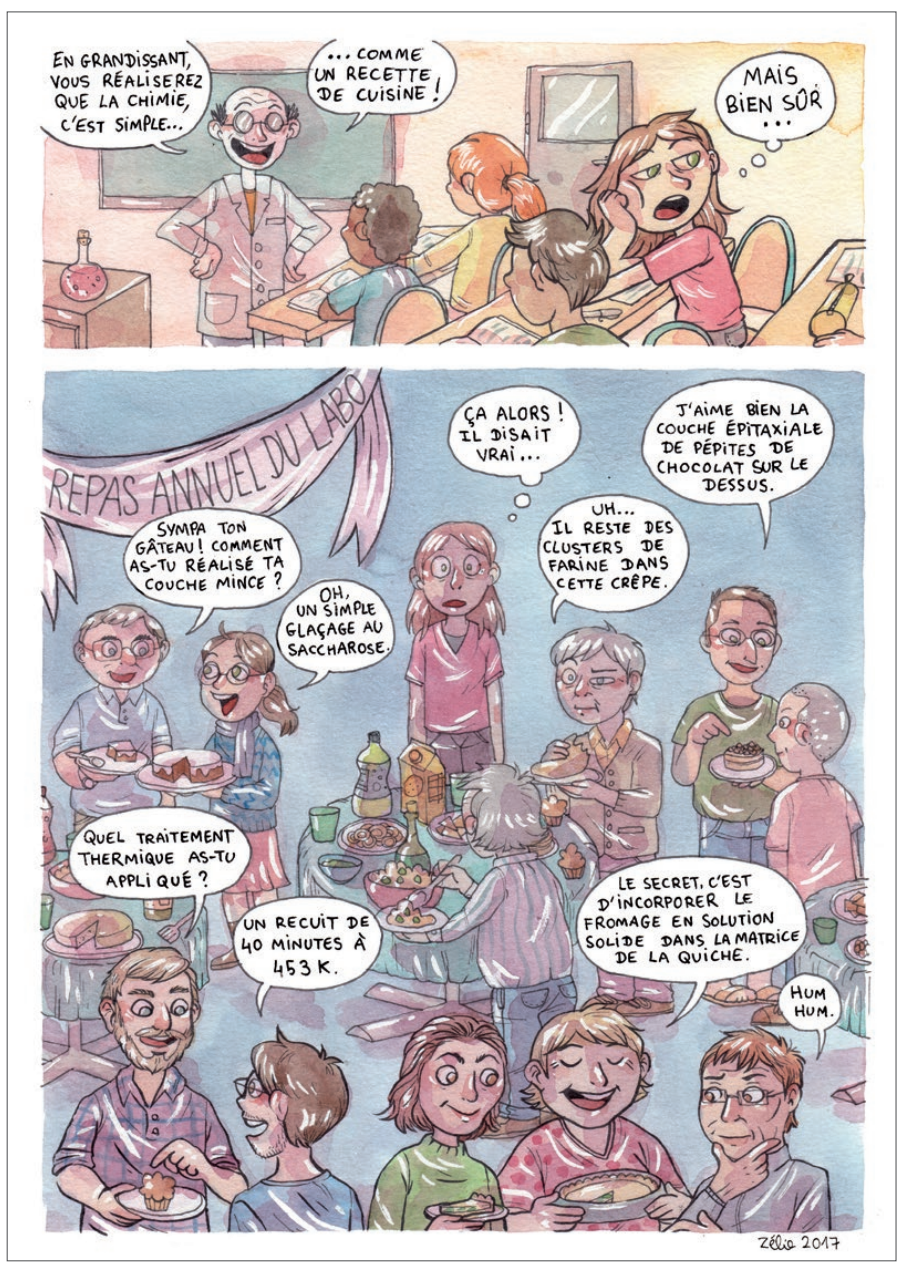

Titre : La Cuisine. Auteur : Zélie Tournoud, doctorante en science des matériaux au SIMAP (Grenoble).

Petite surprise, nous avons découvert plus tard que Zélie tient un blog de dessins : Le Blog de Zélie ! - zelie.fr/blog 


\section{Remise des prix}

La cérémonie de remise de prix s'est tenue à Orsay, pendant le $24^{\mathrm{e}}$ Congrès général de la Société Française de Physique, le 5 juillet 2017.

Mayline Gautié, chargée de communication de la SFP, a présenté le concours et les nombreux cadeaux offerts par l'ensemble des partenaires. Trois d'entre eux ont adressé un message au public au sujet de cette opération : Alain Fontaine, directeur de la Fondation Nanosciences, François Garin, secrétaire général de la Société Chimique de France, et Hélène Snyders, responsable des relations publiques du Théâtre de la Reine Blanche.
Les lauréats ont reçu un lot de la Fondation Nanosciences, d'une valeur totale de $1000 €$, un abonnement d'un an à la version numérique de la revue Science et Avenir, ainsi que de nombreux autres cadeaux (places de spectacle, livres, bandes dessinées...).

La Société Française de Physique et tous ses partenaires remercient chaleureusement l'ensemble des candidat(e)s pour l'immense richesse des œuvres proposées, empreintes d'humour et de créativité, témoignant d'un goût affirmé pour les sciences! La revue Reflets de la Physique en publiera régulièrement dans ses futurs numéros, de sorte que les lecteurs puissent s'en réjouir...

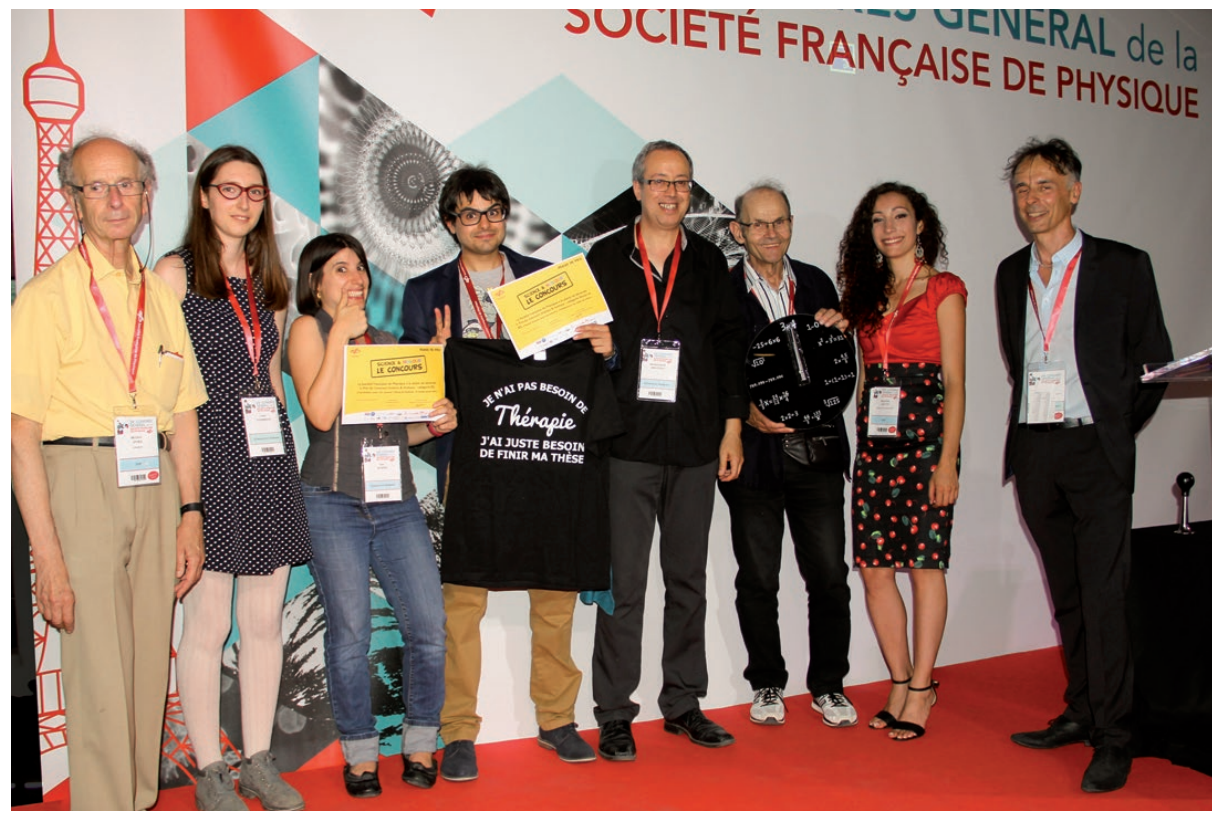

De gauche à droite : Michel Spiro, président de la SFP - Zélie Tournoud, lauréate du vote du public - Ève Redero, lauréate catégorie BD - Benoît Simony, lauréat catégories BD \& dessin - Mohammed Moudjou, lauréat catégorie dessin - Xavier Artru, lauréat catégorie dessin - Mayline Gautié, chargée de communication de la SFP - Olivier Dulieu, président du comité d'organisation du Congrès général 2017.

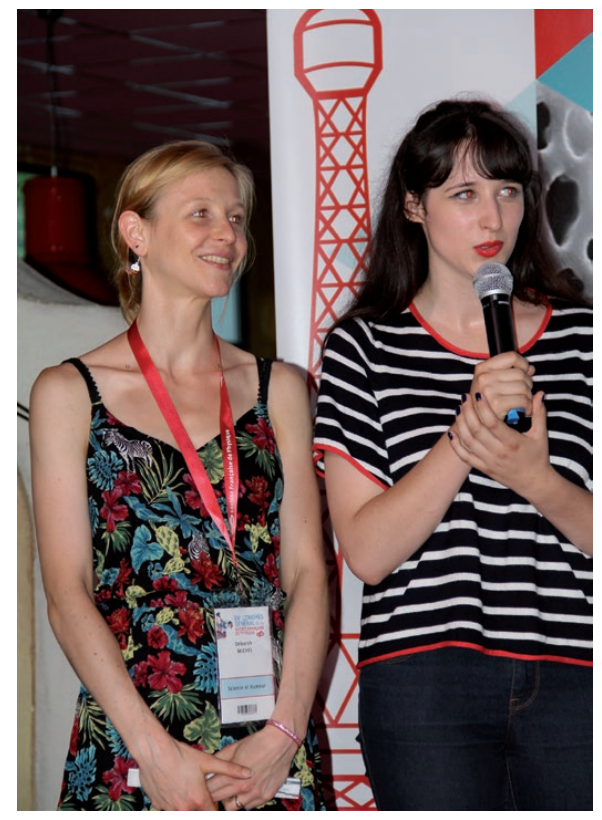

Déborah Michel (à gauche) et Orane Tasky, lauréates de la catégorie photo.

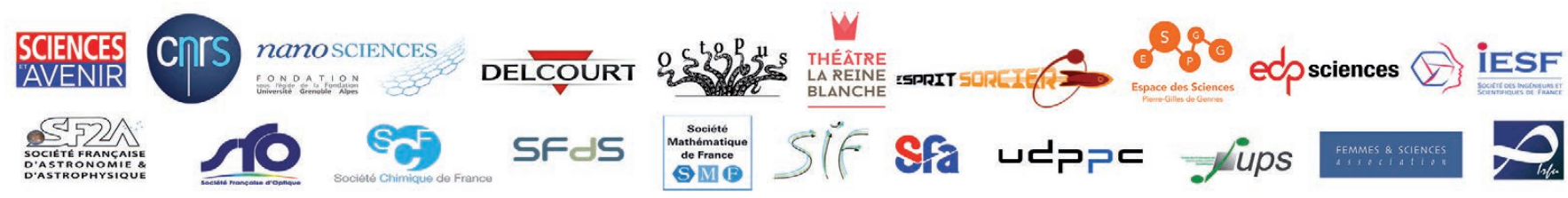

Les logos des partenaires du Concours Science \& Humour. 